\title{
Meromorphic Extensions of Regular Holonomic Distributions
}

\author{
Rolf Källström
}

Department of Theoretical Physics, Royal Institute of Technology, S-10044 Stockholm, Sweden

\begin{abstract}
In this paper we consider distributions of the form $1 / P$ and $\int d y / P(x, y)$, where $P$ is a polynomial. Using results by Kashiwara and Kawai we give fairly accessible proofs that these expressions can be defined as regular holonomic distributions by utilising a meromorphic parameter. We also discuss distributions of the form $\mu / P$ and their direct image $\int \frac{\mu d y}{P(x, y)}$, when one knows that $\mu$ is a regular holonomic distribution. All these distributions are relevant to the study of renormalised Feynman integrals.
\end{abstract}

\section{Introduction}

In [1] Kashiwara and Kawai develop a micro-local theory of holonomic systems with regular singularities. This theory is then applied to show that an analytically renormalised Feynman function satisfies a holonomic system of linear partial differential equations with regular singularities, and as a by-product of their general theory they show that the Feynman functions are all Nilsson class functions [2] (see also [3]). They have also proved that the system of differential equations corresponding to a Feynman function has a Lagrangian characteristic set which is contained in the extended Landau variety in $T^{*}\left(\mathbf{C}^{N}\right)$, for some integer $N$. This implies that the wave front set of the Feynman function (also for complex momenta) is contained in the extended Landau variety. We refer to $[4,5]$ for further discussion about the relevance of regular holonomicity to physics. In this paper we will show that a similar class of expressions, given by rational functions with singularities, also can be defined as regular holonomic distributions. Indeed, it follows from these results that the analytically renormalised Feynman integrals are regular holonomic (see the remark in Sect. 4). We do not claim essential originality because some of our main results are implicitly contained in the work [6]. However, the algebraic theory of regular holonomic $\mathscr{D}$-modules has its special features and is for example presented in [7]. Here we offer reasonably self-contained proofs concerned with existence of meromorphic extensions of certain tempered distributions, which 
culminate in the discovery that these distributions in addition satisfy regular holonomic systems. Let us also remark that regular holonomic $\mathscr{D}$-modules are of particular interest in the theory of algebraic groups and their representations thanks to a far-reaching equivalence of categories. See for instance [7].

Let $P(x, y)$ be a real-valued polynomial, $(x, y) \in \mathbf{R}^{n+m}$. The main purpose of this work is to find a natural definition of the expression

$$
\int_{P>0} \frac{d y}{P(x, y)}
$$

as a tempered regular holonomic distribution. The problem consists of two parts: first define the distribution $\frac{1}{P}$, and then find an extension of the concept of direct images of distributions. Since any reasonable definition of $\frac{1}{P}$ results in a tempered distribution, one can always extend this distribution to projective space along the fibre of integration, $\mathbf{R}^{n} \times \mathbf{R}^{m} \rightarrow \mathbf{R}^{n} \times \mathbf{P}_{m}, \mathbf{P}_{m}=\mathbf{R}^{m} \cup H$, so that the projection $\pi: \mathbf{R}^{n} \times \mathbf{P}_{m} \rightarrow \mathbf{R}^{n},(x, y) \rightarrow x$ is proper. However, the Lebesgue form $d y$ is singular at the hyperplane at infinity $H$, so it is not clear how to define the distribution $\pi_{*}\left(\frac{1}{P}\right)$. The recipe to deal with these problems is to consider the distribution as a member of a family of distributions with an analytic parameter, and establish the existence of meromorphic extensions in which we take the finite part in the Laurent expansion at the interesting value of the parameter.

The contents of the paper are as follows. In Sect. 2 we define the regular holonomic distribution $\frac{1}{P}$, in Sect. 3 we find a regular holonomic direct image of a regular holonomic distribution, and finally in Sect. 4 we discuss the case $\frac{\mu}{P}$ and the problems associated with finding a direct image, when we know that $\mu$ is a regular holonomic distribution on a totally real submanifold of a quasi-projective algebraic variety. We also include appendices about the generalised Stokes formula and the fact that regular holonomic distributions are tempered.

\section{The Distribution $1 / P$}

Let $A_{n+m}$ be the Weyl algebra over $\mathbf{R}^{n+m}$, i.e. the algebra of differential operators with complex-valued polynomial coefficients. Let $\Gamma_{c}\left(\mathbf{R}^{n+m}\right)$ be the test forms, that is $C^{\infty}(n+m)$-forms with compact support. $A_{n+m}$ acts on $\Gamma_{c}$ : for a vector field $\delta$ in $A_{n+m}$ and with $\omega \in \Gamma_{c}$, we define $\delta \cdot \omega=-L_{\delta}(\omega)$, where $L_{\delta}$ is the Lie derivative along $\delta$. It is easy to see that this gives a right action on $\Gamma_{c}$. Consequently, $A_{n+m}$ acts from the left on the distributions $\mathscr{D} b\left(\mathbf{R}^{n+m}\right)$, and it is clear that the space of tempered distributions is a left $A_{n+m}$-submodule of $\mathscr{D} b\left(\mathbf{R}^{n+m}\right)$.

Let $\chi$ be the characteristic function of $\{(x, y): P(x, y)>0\}$. If $\lambda$ is a complex number with $\operatorname{Re} \lambda>0$, we get a locally integrable function, and hence a distribution, $P_{+}^{\lambda}=\chi P^{\lambda} . P^{\lambda}$ also is a generator for the module $A_{n+m}[\lambda] P^{\lambda}$, for which we have a 
functional equation

$$
b(\lambda) P^{\lambda}=Q(\lambda) P^{\lambda+1}, \quad Q(\lambda) \in A_{n+m}[\lambda],
$$

where $b(\lambda) \in \mathbf{C}[\lambda]$ is the Bernstein-Sato polynomial. This is explained in [11].

Proposition 1. The functional equation is also an equality between tempered distributions:

$$
b(\lambda) P_{+}^{\lambda}=Q(\lambda) P_{+}^{\lambda+1} .
$$

Proof. We first let $\operatorname{Re} \lambda>1$ and observe that the action of vector fields on $P_{+}^{\lambda}$ then is the same as the corresponding action in $A_{n+m}[\lambda] P^{\lambda}$. If $\eta$ is a vector field with polynomial coefficients, we get by Federer's generalised Stokes formula (see appendix)

$$
\eta\left(P_{+}^{\lambda}\right)=\chi \eta\left(P^{\lambda}\right)+\eta(P) \delta_{0}(P) P^{\lambda},
$$

where $\delta_{0}$ is the dirac measure. So $\eta\left(\chi P^{\lambda}\right)=\chi \eta\left(P^{\lambda}\right)$ in $\mathscr{D} \mathcal{E}\left(\mathbf{R}^{n+m}\right)$, if $\operatorname{Re} \lambda>1$. It is now clear that the functional equation holds when $\operatorname{Re} \lambda \gg 0$. Furthermore, the right-hand side is analytic when $\operatorname{Re} \lambda>-1-\varepsilon$, for some small number $\varepsilon>0$, so $\lambda \rightarrow P_{+}^{\lambda} \in \mathscr{D} b$ is meromorphic then. One can iterate the functional equation any number of times, which shows that the map $\lambda \rightarrow P_{+}^{\lambda} \in \mathscr{D} b$ is meromorphic in $\mathbf{C}$. Consequently, the functional equation is an equality between meromorphic families of distributions, with poles determined by the polynomial $b(\lambda)$.

Define the tempered distribution $\frac{1}{P}$ by taking the finite part in the Laurent expansion at $\lambda=-1$,

$$
\mu=\text { P.f. }\left(P_{+}^{\lambda}, \lambda=-1\right) .
$$

Let $k$ be the order of the zero of the polynomial $b(\lambda)$ at $\lambda=-1$, i.e. $b(\lambda)=(\lambda+1)^{\ell} b_{1}(\lambda), b_{1}(-1) \neq 0$. We then get the more explicit expression

$$
\mu=\frac{1}{k ! b_{1}(-1)} \sum_{\ell=0}^{k}\left(\begin{array}{l}
k \\
\ell
\end{array}\right) Q_{\ell}(\log P)^{\ell-\ell},
$$

where $Q_{\ell}=\frac{d^{\ell} Q}{d \lambda^{\ell}}(-1)$. Of course, this representation is not unique.

Remark. This method of defining $\frac{1}{P}$ was invented by M. Riesz to solve the Cauchy problem for the wave equation. I. N. Bernstein and I.S. Gelfand [8] and, independently, M. Atiyah [9] used Hironaka's resolution of singularities to establish the meromorphic continuation in the general case. When the existence of the functional equation was proved one also got an elementary proof of the existence of meromorphic continuations [10].

Proposition 2. $\mu$ is a regular holonomic distribution.

In the proof of the proposition we shall need the following: consider the left $A_{n+m}$-module $A_{n+m}[\lambda] P^{\lambda}$ and put

$$
J=\left\{Q \in A_{n+m}: Q P^{\lambda}=\sum_{v>k}(\lambda+1)^{v} Q_{v} P^{\lambda}, Q_{v} \in A_{n+m}\right\} .
$$


From [6] we have

Theorem 3. $A_{n+m} / J$ is a regular holonomic $A_{n+m}$-module.

Proof of the Proposition. First note that the identities in the ideal $J$ are satisfied by the distribution $P_{+}^{\lambda}$, when $\operatorname{Re} \lambda \gg 0$. Indeed, this follows from the generalised Stokes formula, as in the proof of the previous proposition. The equalities for any $\lambda$ follows from meromorphic continuation. By the theorem above it suffices to prove that the left ideal $J$ annihilates $\mu$ in the left $A_{n+m}$-module of tempered distributions. To prove this let $Q \in J, \omega \in \Gamma_{c}$, and $Q^{*}$ be the corresponding action on $\omega$ (the adjoint):

$$
\begin{aligned}
\langle Q \mu, \omega\rangle & =\left\langle\mu, Q^{*} \omega\right\rangle=\text { P.f. }\left(\lambda=-1,\left\langle P_{+}^{\lambda}, Q^{*} \omega\right\rangle\right) \\
& =\text { P.f. }\left(\lambda=-1, \sum_{\nu>k}(\lambda+1)^{\nu}\left\langle P_{+}^{\lambda}, Q^{*} \omega\right\rangle\right)=0,
\end{aligned}
$$

since the order of the pole of $Q P_{+}^{\lambda}$ at $\lambda=-1$ is at most $k$. Thus $J \mu=0$, which completes the proof.

\section{The Direct Image of $1 / P(x, y)$}

Now we construct a distribution theoretic direct image of any regular holonomic distribution $\mu$. In Appendix 2 we discuss the fact that $\mu$ is tempered, i.e. $\mu$ extends to a distribution on projective space $\mathbf{P}_{n}(\mathbf{R})$. Actually this result is not necessary if we are considering the case $\mu=1 / P(x, y)$ as defined above, since it is seen to be tempered by construction. The construction of the direct image goes as follows. Let $\pi: \mathbf{R}^{n+m} \rightarrow \mathbf{R}^{n},(x, y) \rightarrow x$ be the projection and $d y$ be the pull-back of the Lebesgue form on $\mathbf{R}^{m}$. Select any real-valued non-vanishing positive polynomial $r(y)$, such that $r(y) \rightarrow \infty$, when $y \rightarrow \infty$, and introduce the analytic family $\mu_{\lambda}=r^{-\lambda} \mu \in \mathscr{D} b\left(\mathbf{R}^{n+m}\right)$. By Sard's lemma there exist positive constants $C$ and $\delta$ such that $|r(y)| \geqq C(1+|y|)^{\delta}$, and since $\mu$ is tempered we see that the following expression defines a tempered distribution when $\operatorname{Re} \lambda \gg 0$ :

where $\omega \in \Gamma_{c}\left(\mathbf{R}^{n}\right)$.

$$
\lambda \rightarrow\left\langle\pi_{*} \mu_{\lambda}, \omega\right\rangle=\left\langle\mu_{\lambda}, \pi^{*} \omega \wedge d y\right\rangle,
$$

Proposition 5. The map $\mathbf{C} \ni \lambda \rightarrow \pi_{*} \mu_{\lambda} \in \mathscr{D} b\left(\mathbf{R}^{n}\right)$ is meromorphic.

Proof. Since $\mu$ is tempered it is clear that $\lambda \rightarrow \pi_{*} \mu_{\lambda}$ is analytic when $\operatorname{Re} \lambda \gg 0$, with derivate $\lambda \rightarrow \pi_{*}\left(\log (r) \mu_{\lambda}\right)$, for any $\omega \in \Gamma_{c}\left(\mathbf{R}^{n}\right)$. We demonstrate the continuation to any comlex number $\lambda$ by using functional equations in local coordinates for $\mathbf{R}^{n} \times \mathbf{P}_{m}$. Let $U_{0}=\left\{\left[\left(t_{0}, t_{1}, \ldots, t_{m}\right)\right]: t_{0} \neq 0\right\}$ be the embedding of $\mathbf{R}^{m}$ in $\mathbf{P}_{m}$, and $U_{i}=\left\{\left[\left(t_{0}, t_{1}, \ldots, t_{m}\right)\right]: t_{i} \neq 0\right\}, i=1,2, \ldots, m$, which completes an affine covering of $\mathbf{P}_{m}$. By a partition of unity subordinate to this coordinate covering, $1=\Sigma \phi_{i}$, it is sufficient to show that $\lambda \rightarrow\left\langle\mu_{\lambda}, \omega \wedge v_{i}\right\rangle$ is meromorphic when $\omega \in \Gamma_{c}\left(\mathbf{R}^{n}\right)$, and $v_{i}=\phi_{i}(y) d y \in \Gamma_{c}\left(U_{i}\right), i=0,1, \ldots, n$. It is obvious that $\lambda \rightarrow\left\langle\mu_{\lambda}, \omega \wedge v_{0}\right\rangle$ is analytic, so let $1 \leqq i \leqq n$, and $\left(y_{1}, y_{2}, \ldots, y_{m}\right)$ be local coordinates in $U_{i}$, i. e. the transition from $U_{0}$ looks like

$$
y_{1}=\frac{x_{1}}{x_{i}}, \ldots, y_{i-1}=\frac{x_{i-1}}{x_{i}}, y_{i}=\frac{1}{x_{i}}, y_{i+1}=\frac{x_{i+1}}{x_{i}}, \ldots, y_{m}=\frac{x_{m}}{x_{i}} .
$$


In $U_{0} \cap U_{i}$ we have $r(x)=y_{i}^{-d} r_{1}(y)$, where $d$ is the degree of $r(x)$ and $r_{1}(y)$ is another polynomial, and $v_{i}=\phi_{i} y_{i}^{-m-1} d y$. When $\operatorname{Re} \lambda \gg 0$ we have

$$
\left\langle\mu_{\lambda}, \omega \wedge v_{i}\right\rangle=\left\langle y_{i}^{\lambda d-m-1}\left(r_{1}(y)\right)^{-\lambda} \mu, \phi_{i}(y) \omega \wedge d y\right\rangle
$$

Let $\chi_{i}$ be the characteristic function of $\left\{y_{i}>0\right\} . \mu$ is holonomic, so $\lambda \rightarrow \chi_{i} y_{i}^{\lambda d-m-1} \mu \in \mathscr{D} b\left(U_{i}\right)$ is meromorphic by the functional equation for holonomic modules (see [11]), and by the fact that $\partial_{i} \chi_{i}=\delta_{0}\left(y_{i}\right)$. It is easy to see that $r_{1}(y)$ is a unit in $U_{i}$, so $\lambda \rightarrow r_{1}^{-\lambda}$ is analytic and $r_{1}^{-\lambda}(y)$ is real-analytic for any fixed $\lambda$. It follows that the map

$$
\text { C } \lambda \rightarrow\left\langle\chi_{i} \cdot r_{1}(y)^{-\lambda} y_{i}^{\lambda d-m-1} \mu, \phi_{i}(y) \omega \wedge d y\right\rangle
$$

is meromorphic. So $\lambda \rightarrow \pi_{*} \mu_{\lambda}=\sum_{i=0}^{n}\left\langle\mu_{\lambda}, \omega \wedge v_{i}\right\rangle$ also is meromorphic, and by analytic continuation independent of the partition of unity, since any two partitions give the same definition when $\operatorname{Re} \lambda \gg 0$. This completes the proof.

Define the direct image $\pi_{*}\left(\frac{1}{P}\right)$ as the finite part at $\lambda=0$ of $\lambda \rightarrow \pi_{*} \mu_{\lambda}$,

$$
\mu_{*}=\text { P.f. }\left(\lambda=0, \pi_{*} \mu_{\lambda}\right) \text {. }
$$

Obviously, $\mu_{*}$ is a tempered distribution in $\mathbf{R}^{n}$. Note that the definition of $\mu_{*}$ is independent of the choice of the non-vanishing polynomial $r(y)$, if only $r(y) \rightarrow \infty$, when $y \rightarrow \infty$. This can be seen as follows:

$$
\begin{aligned}
\text { P.f. }(\lambda & \left.=0,\left\langle\chi_{i}\left(r_{1}(y)\right)^{-\lambda} y_{i}^{\lambda d-m-1} \mu, \phi_{i}(y) \omega \wedge d y\right\rangle\right) \\
& =\text { P.f. }\left(\lambda=-m-1,\left\langle\chi_{i} \cdot y_{i}^{\lambda} \mu, \phi_{i}(y) \omega \wedge d y\right\rangle\right) .
\end{aligned}
$$

Remark. Of course, this definition of $\int \frac{\chi d y}{P(x, y)}$ coincides with the ordinary direct image when $d y$ is replaced by an $m$-form with compact support.

Theorem 6. $\mu_{*}$ is regular holonomic.

Proof. Let $n_{0}$ be the order of the pole at $\lambda=0$ of $\lambda \rightarrow \pi_{*} \mu_{\lambda}$. Set

where

$$
I=\left\{Q \in A_{n}: Q \in J_{r}+\sum_{1}^{m} \frac{\partial}{\partial y_{v}} P_{v}, P_{v} \in A_{n+m}\right\},
$$

$$
J_{r}=\left\{S \in A_{n+m}: S \mu_{\lambda}=\sum_{\nu>n_{0}} \lambda^{v} S_{v} \mu_{\lambda}, S_{v} \in A_{n+m}\right\} .
$$

$J_{r}$ is an ideal of $A_{n+m}$ which depends on the choice of $r(y)$. Here $Q$ can be considered to act in $A_{n+m}[\lambda] r^{-\lambda} \otimes \mu$, where the distribution $\mu$ is a generator for a regular holonomic module. Take $\omega \in \Gamma_{c}\left(\mathbf{R}^{n}\right)$, so

$$
\left\langle\mu_{*}, \omega\right\rangle=\text { P.f. }\left(\lambda=0,\left\langle\mu_{\lambda}, \pi^{*} \omega \wedge d y\right\rangle\right) \text {. }
$$


Let $Q \in I$, i.e. $Q=S+\sum \frac{\partial}{\partial y_{v}} P_{v} \in A_{n}$, for some $S \in J_{r}, P_{v} \in A_{n+m}$. We get

$$
\begin{aligned}
\left\langle Q \mu_{*}, \omega\right\rangle & =\left\langle\mu_{*}, Q^{*} \omega\right\rangle=\text { P.f. }\left(\lambda=0,\left\langle\mu_{\lambda}, \pi^{*}\left(\mathrm{Q}^{*} \omega\right) \wedge d y\right\rangle\right) \\
& =\text { P.f. }\left(\lambda=0,\left\langle\mu_{\lambda},\left(\pi^{*}\left(\mathrm{~S}^{*}-\sum_{1}^{m} P_{v}^{*} \frac{\partial}{\partial y_{v}}\right) \omega\right) \wedge d y\right\rangle\right) \\
& =\text { P.f. }\left(\lambda=0,\left\langle\mu_{\lambda},\left(S^{*} \pi^{*} \omega\right) \wedge d y-\left(\sum_{v=1}^{m} P_{v}^{*} \mathbf{R}^{n+m} \pi^{*}(\omega)\right) \wedge d y\right\rangle\right) .
\end{aligned}
$$

Here $A_{n}$ is considered as a subring of $A_{n+m}$. We have, for any operator $S$ in $A_{n+m}$, $S d y=S^{0} d y$, where $S^{0}$ is the zero-order part of $S$; here it is important that the ideal $J_{r}$ consists of global sections along the fibre of integration and that $d y$ is translation invariant, meaning $\delta(d y)=0$ when $\delta$ is a vector field. It follows that $\left(S^{*} \pi^{*} \omega\right) \wedge d y$ $=S^{*}\left(\pi^{*} \omega \wedge d y\right)$. Since $\frac{\partial}{\partial y_{v}} \pi^{*}(\omega)=0$, we find

$$
\begin{aligned}
\left\langle Q \mu_{*}, \omega\right\rangle & =\text { P.f. }\left(\lambda=0,\left\langle\mu_{\lambda}, \mathrm{S}^{*}\left(\pi^{*} \omega \wedge \text { dy }\right)\right\rangle\right) \\
& =\text { P.f. }\left(\lambda=0, \sum_{v>n_{0}} \lambda^{v}\left\langle\mu_{\lambda}, S_{v}^{*}\left(\pi^{*} \omega \wedge d y\right)\right\rangle\right)=0 .
\end{aligned}
$$

The last equality follows because the order of the pole at $\lambda=0$ of the map

$$
\lambda \rightarrow\left\langle Q\left(\pi_{*} \mu_{\lambda}\right), \omega\right\rangle=\left\langle\pi_{*}\left(\mu_{\lambda}\right), Q^{*} \omega\right\rangle, \quad Q \in A_{n}
$$

is at most $n_{0}$. Hence we have proved that $I$ annihilates $\mu_{*}$. The theorem now is a consequence of the following result.

Theorem 7. $A_{n} / I$ is a regular holonomic $A_{n}$-module.

Sketch of Proof. In general let $M$ be a regular holonomic $A_{n+m}$-module. In the category of modules over differential operators the projection $\pi: \mathbf{C}^{n+m} \rightarrow \mathbf{C}^{n}$ gives the direct image $\pi_{+} M$. The construction of $\pi_{+} M$ is explained in detail in [7]. Here we can use that $\mathbf{C}^{n+m}$ is affine and directly conclude that $\pi_{+} M$ can be identified with the complex of left $A_{n}$-modules obtained by the usual Koszul-complex $\mathscr{K}\left(M ; \frac{\partial}{\partial y_{1}}, \ldots, \frac{\partial}{\partial y_{m}}\right)$ shifted in degree $-m$. In particular the left $A_{n}$-module $M / \sum_{1}^{m} \frac{\partial}{\partial y_{v}} M$ is a cohomology module of $\pi_{+} M$. By the results of Bernstein as explained in Sect. 12, Chap. 7 of [7] $\pi_{+}$preserves regular holonomicity. Hence $M / \sum_{1}^{m} \frac{\partial}{\partial y_{v}} M$ is a regular holonomic $A_{n}$-module. In particular let $M=A_{n+m} / J_{r}$. Using results from [6] it follows that $M$ is a regular holonomic $A_{n+m}$-module. Now it is clear that $A_{n} / I$ is a submodule of $M / \sum_{1}^{m} \frac{\partial}{\partial y_{v}} M$, and thus regular holonomic. 


\section{Extension of the Results to Distributions of the Form $\frac{\mu}{P}$}

In this section we discuss possible extensions of the preceding results. Let $M$ be a totally real submanifold of a quasi-projective manifold $X \rightarrow \mathbf{P}_{m}(\mathbf{C})$. On $X$ there exists the sheaf of rings $\mathscr{D}_{X}$ of differential operators with algebraic coefficients and one can restrict the elements of $\mathscr{D}_{X}$ to $M$. Assume that we are given a distribution of finite order $\mu$ on $M$ which satisfies a regular holonomic system $\mathscr{L} \subset \mathscr{D}_{X} \otimes_{\mathbf{C}} A_{n}(\mathbf{R})$. Here $\mathscr{L}$ is a sheaf of ideals and $X$ is equipped with the Zariski topology. So $Q \mu=0$ if $Q \in \mathscr{L}(U)$, where $U$ is Zariski open. Let $P(x, y)$ be an affine function in $\mathbf{R}^{n} \times X$, such that the restriction to $\mathbf{R}^{n} \times M$ is real-valued. Since $\mu$ is of finite order, it follows from Sard's theorem that we can define the distribution $P_{+}^{\lambda} \mu$ on $\mathbf{R}^{n} \times M$ when $\operatorname{Re} \lambda \gg 0$. For an affine neighbourhood $U \subset X$ we have in $\mathbf{R}^{n} \times(U \Phi M)$ a functional equation for holonomic modules [11]

$$
\mathrm{b}(\lambda) P^{\lambda} \mu=Q(\lambda) P^{\lambda+1} \mu,
$$

where $Q(\lambda)$ is a polynomial in $\lambda$ with coefficients in $A_{n+m}$. By the Stokes formula this equation also holds in the distribution sense

$$
b(\lambda) P_{+}^{\lambda} \mu=Q(\lambda) P_{+}^{\lambda+1} \mu .
$$

It follows that the map $\lambda \rightarrow P_{+}^{\lambda}$ is meromorphic, whence we can define

$$
\frac{\mu}{P}=\text { P.f. }\left(\lambda=-1, P_{+}^{\lambda} \mu\right)
$$

Kashiwara and Kawai [6] have proved the following parallel to Theorem 3. Consider the sheaf of ideals

$$
J=\left\{Q \in \mathscr{D}_{X} \otimes_{\mathbf{C}} A_{n}: Q P^{\lambda} \otimes \mu=(\lambda+1)^{k} Q(\lambda) P^{\lambda} \mu\right\},
$$

where $Q(\lambda)$ is a polynomial with coefficients in $\mathscr{D}_{X} \otimes_{\mathbf{C}} A_{n}(\mathbf{R})$. Then we have

Theorem 8. $\mathscr{D}_{X} \otimes_{\mathbf{C}} A_{n}(\mathbf{R}) / J$ is a sheaf of regular holonomic modules.

As in the proof of Proposition 2 we then can use this to prove that $\mu / P$ is regular holonomic.

The problem of naturally defining $\pi_{*}\left(\frac{\mu}{P}\right)$ as a regular holonomic distribution is one of existence of global sections and an invariant integration form. Suppose there exists a nonvanishing volume form $d y$ on $M$ that is translation invariant, meaning $\delta(d y)=0$ when $\delta$ is a vector field, and such that $d y$ has tempered growth at $\partial M$. Furthermore, assume that there exists an affine function $r(y), y \in X$ such that $r(y) \neq 0$ when $y \in M$ and $r(y)=0$ when $y \in \partial M$. One can then prove a parallel to Proposition 12 in Appendix 2, so $\mu$ is tempered at $\partial M \subset \mathbf{P}_{m}(\mathbf{C})$, whence one can use Sard's lemma and functional equations in affine charts to prove that

$$
\lambda \rightarrow\left\langle\pi_{*} r^{\lambda} \frac{\mu}{P}, \omega\right\rangle=\left\langle r^{\lambda} \frac{\mu}{P}, \pi^{*} \omega \wedge d y\right\rangle
$$

defines a meromorphic family of distributions in $\mathbf{R}^{n}$. Here $\pi: \mathbf{R}^{n} \times M \rightarrow \mathbf{R}^{n}$ is the projection and $\omega \in \Gamma_{0}\left(\mathbf{R}^{n}\right)$. In order to carry through a proof similar to the proof of 
Theorem 6 we need that the ideal $J$ above (where $P^{\lambda}$ is replaced by $r^{\lambda}, \mu$ by $\frac{\mu}{P}$, and $\lambda+1$ by $\lambda)$ contains enough global sections so that one can prove an analogue of Theorem 7. We believe that the conditions for this procedure are fulfilled when $M$ is an algebraic group, in which case $d y$ is the Haar measure and the vector fields in $\mathscr{D}_{X}$ are left invariant.

Remark. It is possible to prove that the above procedure is applicable in the case $M=\mathbf{R}^{m}$. Knowing this it is easy to see that the analytically renormalised Feynman integrals are regular holonomic by iterating the construction when $P$ is the KleinGordon polynomial in various subsets of coordinates in $\mathbf{R}^{m}$, and $\mu$ in the first step is the dirac function corresponding to the conservation of momenta in $\mathbf{R}^{n} \times \mathbf{R}^{m}$ at the vertices in the Feynman diagram. Note that

$$
(P(x)+i 0)^{\lambda}=P_{+}^{\lambda}(x)+e^{\pi i \lambda} P_{-}^{\lambda}(x),
$$

which is defined for any real-valued polynomial $P$ and any $\lambda \in \mathbf{C}$ by taking the finite part in a Laurent expansion of the right-hand side. Here $P_{-}^{\lambda}(x)=|P(x)|^{\lambda}$, when $P(x)<0$, and zero elsewhere. In particular we see that the Feynman propagator is a linear combination of two regular holonomic distributions and hence regular holonomic.

\section{Appendix 1. The Generalised Stokes Formula}

The Stokes formula is usually proved for sets $\Omega$ with boundary $\partial \Omega$ of class $C^{1}$, that is $\partial \Omega$ is a differentiable manifold so that the implicit function theorem applies and $\partial \Omega$ locally can be straightened out. Often this is not enough for applications, and it is easy to see that one can allow some small singular subset of $\partial \Omega$. Actually, the following quite optimal generalisation is not hard to prove and is contained in a theorem by $\mathrm{H}$. Federer [12]

Theorem 9. Let $\Omega$ be a Lebesgue measurable set in $\mathbf{R}^{n}$ with boundary $\partial \Omega=\Gamma \cup \Sigma$, where $\Gamma$ is of class $C^{1}$ and the Hausdorff dimension of $\Sigma, \operatorname{dim}_{H} \Sigma<n-1$. Suppose that the area measure $d S$ on $\Gamma$ extends to a measure on $\mathbf{R}^{n}$ such that $\Sigma$ is a null set with respect to $d S$. Let $\chi_{\Omega}$ be the characteristic function of $\Omega$.

Then $\partial_{j} \chi_{\Omega}=n_{j} d S$, where $n_{j}$ is the exterior normal field on $\Gamma$.

That $d S$ extends to a measure on $\mathbf{R}^{n}$, and in particular to $\partial \Omega$, such that $\Sigma$ is a null set essentially means that $\Gamma$ locally has finite area.

Proof. Since $d S$ extends to a measure on $\mathbf{R}^{n}$ it suffices, by a partition of unity, to prove the theorem for sets $\omega \cap \Omega$, where $\omega$ is an open relatively compact set. If $\omega \cap \Sigma=\emptyset$ the regular Stokes formula applies. Suppose $\omega \cap \Sigma \neq \emptyset$. Let $\pi: \mathbf{R}^{n} \rightarrow \mathbf{R}^{n-1}$ be the projection on the plane $x_{j}=0$. Select a sequence $\phi_{v}$ of $C^{\infty}$-functions in $\mathbf{R}^{n-1}$ such that, for any $v, \phi_{v}=0$ in a neighborhood of $\pi(\Sigma \cap \omega)$. We can assume that $0 \leqq \phi_{\nu} \leqq 1$ holds and $\phi_{v} \rightarrow 1, j \rightarrow \infty$, uniformly on compact subsets of $\mathbf{R}^{n-1} \backslash \pi(\Sigma \cap \omega)$. By the usual Stokes formula we then have the following identity between distributions in $\omega \cap \Omega$ :

$$
\partial_{j}\left(\phi_{v} \chi\right)=\phi_{v} \partial_{j}(\chi)=\phi_{v} n_{j} d S
$$


since $\phi_{v}$ does not depend on $x_{j}$. Now, since $\operatorname{dim}_{H} \Sigma<n-1$ implies $\pi(\Sigma)$ is a Lebesgue null set in $\mathbf{R}^{n-1}$ it follows that $\pi^{-1}(\pi(\Sigma)) \cap \omega$ is a null set in $\mathbf{R}^{n}$ and $\phi_{v} \partial_{j}(\chi) \rightarrow \partial_{j}(\chi), v \rightarrow \infty$, almost everywhere. Finally one verifies that $\phi_{v} \rightarrow 1$ almost everywhere with respect to $n_{j} d S$, which proves the theorem.

In the proof of the existence of functional equations for distributions we need the following theorem due to Whitney [12].

Theorem 10. Let $P(x)$ be a real-valued polynomial in $\mathbf{R}^{n}$. Then the set $\Omega=\{x: P(x)>0\}$ satisfies the conditions for the generalised Stokes formula.

We have $\partial_{j} \chi(P)=\left(\partial_{j} P\right) \delta_{0}(P)$, where $\delta_{0}$ is the dirac measure.

\section{Appendix 2. Regular Holonomic Distributions are Tempered}

In this appendix we discuss the following two basic results

Proposition 11. A regular holonomic distribution $\mu \in \mathscr{D} b\left(\mathbf{C}^{n}\right)$ is tempered.

Propsotiion 12. A regular holonomic distribution $\mu \in \mathscr{D} b\left(\mathbf{R}^{n}\right)$ is tempered.

Proposition 11 is due to Kashiwara [13], but here we offer a sketch of a proof in the spirit of [7], as explained to me by J.-E. Björk. Although we do not give a complete proof of Proposition 12 it might be instructive to see how it is proved in a special case. The general case is covered by Proposition 11 and the work in [14] about the tempered Hom-functor which concerns comparison theorems between regular holonomic $\mathscr{D}$-modules of hyperfunctions and distributions respectively.

Proof of Proposition 11. By Theorem 9.1 in Chap. 7 of [7] every sheaf of coherent regular holonomic $\mathscr{D}$-modules on $\mathbf{P}_{n}(\mathbf{C})$ is generated by global sections. Using this fact we easily reduce the proof to the following case: there exists a coherent sheaf of left ideals $\mathscr{L}$ of $\mathscr{D}_{\mathbf{P}_{n}}$ such that $\mathscr{D}_{\mathbf{P}_{n}} / \mathscr{L}$ is a sheaf of regular holonomic $\mathscr{D}_{\mathbf{P}_{n}}$-modules, and moreover identifying $\mathbf{C}^{n}$ with $\mathbf{P}_{n}(\mathbf{C}) \backslash H$, where $H$ is a hyperplane, $\left.\mathscr{L}\right|_{\mathbf{C}^{n}}$ is equal to the sheaf of left ideals in $\mathscr{D}_{\mathbf{C}^{n}}$ which annihilates $\mu$. Next it is well-known that $\Gamma\left(\mathbf{P}_{n}, \mathscr{L}\right)$, i.e. the global sections of $\mathscr{L}$ over $\mathbf{P}_{n}$, is a left ideal which after restrictions to $\mathbf{C}^{n}$ gives a left ideal of the Weyl algebra $A_{n}(\mathbf{C})$ which is equal to the annihilator of $\mu$ in the cyclic $A_{n}$-module generated by $\mu$. It remains to see that $\mu$ is tempered. To prove this we use the main result in Kashiwara's work [13]. Namely by Proposition 5 in [13] the sheaf

$$
\operatorname{Hom}_{\mathscr{D}_{\mathbf{P}_{n}}}\left(\mathscr{D}_{\mathbf{P}_{n}} / \mathscr{L}, \mathscr{D} b_{\mathbf{P}_{n}}\right)
$$

contains a global section $\gamma$ which is an extension of $\mu$.

Sketch of Proof of Proposition 12 when $\operatorname{dim}(\operatorname{supp}(\mu))=n$. We indicate how the proof can be reduced to the corresponding result for distributions in $\mathbf{C}^{n}$. First we have the natural embeddings $\mathbf{R}^{n} \rightarrow \mathbf{P}_{n}(\mathbf{R}) \rightarrow \mathbf{P}_{n}(\mathbf{C})$, where $\mathbf{P}_{n}$ denotes the real and complex projective space respectively. Working in an affine chart at a hyperplane at infinity the distribution $\mu$ is defined outside a hyperplane $H$ in $\mathbf{R}^{n}$. We want to be sure that $\mu$ has tempered growth at this hyperplane. To do this we introduce the ideal $L_{\mu}=\left\{Q \in A_{n}(\mathbf{C}): Q \mu=0\right\}$ and consider the set $S_{\mu}=\left\{z \in \mathbf{C}^{n}: \exists \zeta \neq 0, \zeta \in \mathbf{C}^{n}\right.$, such that $\sigma(Q)(z, \zeta)=0$ for all $\left.Q \in L_{\mu}\right\}$, where $\sigma(Q)(z, \zeta)$ is the principal symbol of $Q$. 
One can prove that $S_{\mu}$ is an algebraic variety. That $\mu$ is holonomic implies $\operatorname{dim}_{\mathbf{C}} S_{\mu} \leqq n-1$, so in particular $S_{\mu} \cap \mathbf{R}^{n}$ is contained in an algebraic hypersurface. Let $\Omega$ be some connected component of $\mathbf{R}^{n} \backslash S_{\mu}$. Since the analytic wave front set $W F_{A}(\mu) \subset\left(S_{\mu} \cap \mathbf{R}^{n}\right) \times \mathbf{R}^{n}$ and $\operatorname{dim}(\operatorname{supp}(\mu))=n$, it follows that $\mu$ determines a nonvanishing real-analytic function in $\Omega$. One can prove that, starting from the germ at any $x_{0} \in \Omega, \mu$ builds up a multi-valued analytic function $F$ in $\mathbf{C}^{n} \backslash S_{\mu}$, such that $L_{\mu} F=0$. We refer to Chap. 5, Sect. 7 in [11] for more details. Now, since $A_{n}(\mathbf{C}) / L_{\mu}$ is a regular holonomic left $A_{n}(\mathbf{C})$-module it follows from a general theorem in [1] that $F$ belongs to the Nilsson class, which in particular implies that the real-analytic function $\mu$ in $\Omega$ has tempered growth at $S_{\mu} \cap H \subset S_{\mu} \cap \mathbf{R}^{n}$, as asserted.

Acknowledgements. I want to thank Prof. Jan-Erik Björk for giving me the problem that is treated in this work. Without his guidance I would not have got far in solving it. I also want to thank Prof. T. Kawai for some useful comments.

\section{References}

1. Kashiwara, M., Kawai, T.: On holonomic systems of micro-differential equations. III. Systems with regular singularities. Publ. RIMS, Kyoto Univ. 17, 813-979 (1981)

2. Kashiwara, M., Kawai, T.: Holonomic character and local monodromy structure of Feynman integrals. Commun. Math. Phys. 54, 121-134 (1977)

3. Kashiwara, M., Kawai, T., Oshima, T.: A study of integrals by micro-differential equations. Commun. Math. Phys. 60, 97-130 (1978)

4. Kashiwara, M., Kawai, T.: The theory of holonomic systems with regular singularities and its relevance to physical problems. Lecture Notes in Physics, Vol. 126, pp. 5-20. Berlin, Heidelberg, New York: Springer 1980

5. Kawai, T., Stapp, H.: On the regular holonomic character of the $S$-matrix and micro-local analysis of unitarity-type integrals. Commun. Math. Phys. 83, 213-242 (1982)

6. Kashiwara, M., Kawai, T.: On the characteristic variety of a holonomic system with regular singularities. Adv. Math. 32, 163-184 (1979)

7. Borel, A. et al.: Algebraic $D$-modules. New York, London: Academic Press 1987

8. Bernstein, I.N., Gelfand, I.S. : Meromorphy of the function $P$. Funz. Analysis. Akad. Nauk CCCR, 3, 84-86 (1969)

9. Atiyah, M.F.: Resolution of singularities and divisions of distributions. Commun. Pure Appl. Math. 23, 146-150 (1970)

10. Bernstein, I.N.: The analytic continuation of generalised functions with respect to a parameter. Funz. Analysis. Akad. Nauk CCCR, 6, 36-40 (1972)

11. Björk, J.-E.: Rings of differential operators. North-Holland, Vol. 21 (1979); see also Ehlers, F.: "The weyl algebra", in [7]

12. Federer, H. : Geometric measure theory, p. 478. Berlin, Heidelberg, New York : Springer 1969

13. Kashiwara, M.: Regular holonomic $D$-modules and distributions on complex manifolds. Adv. Stud. Pure Math. 8, 199-206 (1986)

14. Kashiwara, M.: The Riemann-Hilbert problem for holonomic systems. Publ. RIMS, Kyoto Univ. 20, 319-365 (1984)

Communicated by H. Araki

Received February 21, 1989; in revised form April 22, 1989 